\title{
Knowledge Construction and Representation in E- Learning using Semantic Web Techniques
}

\author{
Jale Narimisaei \\ Department of Computer, \\ Faculty of Engineering \\ Shahid Chamran University \\ Ahvaz, Iran
}

\author{
Bita Shadgar \\ Department of Computer, \\ Faculty of Engineering \\ Shahid Chamran University \\ Ahvaz, Iran
}

\author{
Alireza Osareh \\ Department of Computer, \\ Faculty of Engineering \\ Shahid Chamran University \\ Ahvaz, Iran
}

\begin{abstract}
The reason for the growth of e-learning that it provides a convenient and efficient learning environment and practical utilities at anytime and anywhere, so that learners and teachers realize their educational activities with less effort, time and money. In recent years utilization of semantic web techniques for knowledge construction and representation in E-learning gained so much attention. This article presents a system for automatically learning knowledge construction and representation in e-learning which is based on the concept map, RDF data modeling and ontology languages. These concepts can show the mentioned knowledge better and also they convey the meaning. In the proposed method, DBpedia knowledge base is used for extracting learning concepts, also the concept map is applied for creating a graph of concepts and relations between them and editor tools of ontology like protégé are providing a knowledge base of concept map in the format of OWL. The proposed method is developed for creating knowledge base from existing concepts in fuzzy logic domain. Among the advantages of the constructed knowledge base we can mention the meaningfulness, its sharing and reusability in all the learning systems.
\end{abstract}

\section{Keywords}

E-Learning, Knowledge Representation, Knowledge Construction, Ontology, Semantic Web.

\section{INTRODUCTION}

Within a decade, the Internet has become a pervasive medium that has changed completely, and perhaps irreversibly, the way information and knowledge are transmitted and shared throughout the world. The education community has not limited itself to the role of passive actor in this unfolding story, but it has been at the forefront of most of the changes [1]. This is a form of computer-aided instruction virtually independent of a specific location and any specific hardware platform [2]. The reason for the growth of is that it provides a convenient and efficient learning environment and practical utilities at anytime and anywhere [3]. Learners and teachers realize their pedagogic activities with less effort, time and money [4]. The role of student is inactive in the traditional learning but she/he has an active role in e-learning. This kind of learning provides a new autonomous-learning environment that combines (1) Multi-media content, (2) Collaboration among learners, and (3) Computer-supported learning [5]. One of the hottest topics in recent years in the AI community, as well as in the Internet community, is the Semantic Web [6]. It has gained the attention of many researches in e-learning field. The semantic web aims at adding semantic information to web contents in order to create an environment in which software agents will be capable of doing tasks efficiently [7]. The basic properties of the semantic web allow for meeting e- learning requirements: quickness, just-in-time and pertinent learning [8]. Today the use of semantic web techniques like concept map, $\mathrm{RDF}^{1}$ data modeling and ontology languages such as $\mathrm{OWL}^{2}$ to construct and represent concepts and knowledge bases in e-learning has improved over the world. Concept mapping can be seen as a first step in ontology building and can also be used flexibly to represent knowledge structure for meaningful learning [9]. A concept map is a graphical representation where nodes represent concepts and links represent the relationships between concepts [10]. RDF is a simple metadata representation framework, using URIs to identify Web-based resources and a graph model for describing relationships between resources. Several syntactic representations are available, including a standard XML format [11]. Regardless of the representation syntax, RDF models use traditional knowledge representation techniques in order to provide better semantic interoperability [6]. RDF Schema is a simple type modeling language for describing classes of resources and properties between them in the basic RDF model. It provides a simple reasoning framework for inferring types of resources [11]. Indeed it defines the vocabulary of an RDF model [6]. Ontologies have become the de-facto standard knowledge representation technology after the emergence of the semantic web. In a web context, ontologies provide a shared understanding of a domain. Such sharing is needed to avoid terminological differences [8]. Ontologies are an important means in semantic web to achieve the semantic interoperability among heterogeneous distributed systems [12]. Software agents apply the knowledge represented in the Ontologies during their intelligent decision making process [13]. Ontologies for elearning can be created in various methods, so that they use from a different vocabulary to describe their terms. This way gives a valid tool to the learning process [14]. In this article, we use semantic web techniques like concept map, ontology and RDF data modeling for knowledge construction and representation from DBpedia knowledge base. The use of these techniques is a suitable method to represent knowledge and semantics, compared to other techniques like HTML ${ }^{3}$ and $\mathrm{XML}^{4}$, because XML documents include syntax and HTML language is used to mark informations. Both of them include no semantics and it is a defect. In this study, we use web services and SPARQL query language to extract desired concepts from DBpedia knowledge base. We develop this method for learning concept of fuzzy logic course. This paper is organized as follows. Second part is a review on learning systems based on semantic web techniques that some of them

\footnotetext{
${ }^{1}$ Resource Description Framework

${ }^{2}$ Web Ontology Language

${ }^{3}$ Hyper Text Markup Language

${ }^{4}$ Extensible Markup Language
} 
use ontology languages and RDF data modeling to represent their domain knowledge, third part describes how to extract concepts from DBpedia knowledge base, fourth part describes how to construct concept maps from extracted concepts in third part, fifth part expresses how to construct ontology in protégé editor and finally sixth part is conclusion and future work.

\section{OVERVIEW OF E-LEARNING SYSTEMS BASED ON SEMANTIC WEB TECHNIQUES}

The existence of semantic in ontology languages and the advantage of visualization of concept maps cause several researchers study on these issues.

Reference [13] reports on the advances of the Semantic ELearning Agent (SEA) project, whose objective is to develop virtual student advisors that render support to university students in order to successfully organize and perform their studies. Advisors should be able to answer questions regarding the regulations of study for example; does a student possess all requirements to participate in an examination or a course?, is a student allowed to register for his/her thesis?. They use ontology languages such as DAML + OIL and OWL to construct their knowledge base. They have been developed two different Ontologies for their e-learning agents: on the one hand, ontology describing the organization structure of a university department, on the other hand, an ontology holding the knowledge about a specific user of the system. Reference [6] presents the Semantic Web-Based model for their elearning system. This model include various services and tools in the context of a semantic portal, such as: course registration, uploading course documents and student assignments, interactive tutorial, announcements, useful links, assessment, and simple semantic search. A metadata-based ontology is introduced for this purpose and added to their model. The OWL language is used to develop their Ontologies. In these Ontologies, the actual resources and properties specified in the RDF models are defined. Reference [12] applies ontology based Multi-agent approach to provide different services like course registration and scheduling in academic institutions. This system leverages the power of both ontology and Multi-agent system. They are developed semantic web agents for different parts of university teaching environment like course agent, faculty agent, student agent, so on. Agents are communicating via one of the most popular agent communication language, $\mathrm{KQML}^{5}$. The ontology which represents the domain knowledge is passed as a parameter between agents. Their ontology language is OWL. The combined use of Ontologies and multi-agent technologies enable sharing of heterogeneous, autonomous knowledge sources in a capable, adaptable and extensible manner. Therefore agents of different academic institutions can communicate each other and ask any question. For example; one can ask other: what courses are taught in what department?. References [8, 15] use OWL ontology to product assessment system. This system control Students' skills and send a feedback to tutor. For it, each student is prompted to express his/her beliefs by building her/his own disciplinerelated ontology and then it is compared to a reference one. The analysis of students' mistakes allows to propose them personalized recommendations and to improve the course materials in general. They present a semantic web technologies-based multi-agent system that allows to

\footnotetext{
${ }^{5}$ Knowledge Query and Manipulation Language
}

automatically control students' acquired knowledge in elearning frameworks. The aim of [9] is to automatically construct e-learning domain concept maps by applying text mining techniques from academic articles. In order to construct the concept map for e-Learning domain, they use the "keywords" listed in journal articles as the "nodes" to represent concepts and the "relation strengths" between any two keywords appeared in the articles as the "links" to represent concept relationships. The "relation strength" between two keywords is measured by the distance of the two keywords appeared in the articles. The constructed concept maps can provide a useful reference for researchers, who are new to the e-Leaning field, to study related issues, for teachers to design adaptive learning materials, and for learners to understand the whole picture of e-Learning domain knowledge. Reference [16] presents an e-learning management system with metadata that serves as a general template for the situation of Thai learners. The system comprises ontology in OWL language for the elearning process, such as course syllabus, teaching methods, learning activities, and learning styles.

\section{CONCEPTS EXTRACTION FROM DBPEDIA KNOWLEDGE BASE}

The DBpedia project is a community effort to extract structured information from Wikipedia and to make this information accessible on the Web. The resulting DBpedia knowledge base currently describes over 2.6 million entities. For each of these entities, DBpedia defines a globally unique identifier that can be dereferenced over the Web into a rich RDF description of the entity, including (1) human-readable definitions in 30 languages, (2) relationships to other resources, (3) classifications in four concept hierarchies, and (4) various facts as well as data-level links to other Web data sources describing the entity [17].

In this knowledge base, Resources are assigned a URI according to the pattern http://dbpedia.org/resource/Name, where Name is taken from the URL of the source Wikipedia article, which has the form http://en.wikipedia.org/wiki/Name [17]. For example, we can access to fuzzy logic concept information by using the pattern http://dbpedia.org/resource /Fuzzy_logic. In this study, we use this knowledge base to extract our desired concepts in domain e-learning. We develop our system on fuzzy logic course, which fuzzy logic concept is a course category and fuzzy logic sub-category and related concepts with it are course units in our system. In [3] have been used course category and course unit terms for neural network course. They have built their desired concepts themselves. They did not use DBpedia Knowledge base.

We use two methods to get these concepts. First method is direct access to DBpedia knowledge base by SPARQL endpoint to extract fuzzy logic concept and its sub-category like fuzzy set, fuzzy number, etc. There are four access mechanisms to DBpedia knowledge base. One of them is SPARQL Endpoint. SPARQL endpoint is provided for querying the DBpedia dataset. Client applications can send queries over the SPARQL protocol to this endpoint at http://dbpedia.org/sparql and get results in a desired format like RDF/XML [17]. We use this Endpoint to access to desired concepts in fuzzy logic context. Second method is to get related concepts with fuzzy logic by using of web services that [18] have implemented. In [18] is implemented a hybrid ranking algorithm, DBpediaRanker. It can be applied to access to related resources to a resource. This algorithm is 
used to rank recourses in DBpedia. It explores the DBpedia graph and queries external information sources in order to compute a similarity value for each pair of resources reached during the exploration. They implement the web services to access to this algorithm. We use them.

\section{CONCEPT MAP CONSTRUCTION}

After extracting desired concepts from DBpedia, we construct concept maps from them because it shows concepts and relations between them well. Concept maps are more suitable to represent domain ontology [9]. Figure 1 shows one of created concept maps by IHMC CampsTools. It is about fuzzy logic category and its sub-category. The concepts like Tnorm, Degree_of_truth and etc. are in category of fuzzy_logic as Figure 1 shows. In this study, the relations like those in DBpedia are used, because they are global and the same in other concept maps and ontologies in this knowledge base. Also it causes our knowledge base becomes global. For example "dcterms:subject" relation expresses which concepts like T-norm exist in fuzzy logic category.

\section{ONTOLOGY CONSTRUCTION}

In this section, we construct our ontology from the concept maps that we created in before section. We use protégé editor to construct and represent our knowledge. Protégé is ontology's editor. In ontology, classes are nodes in concept map and properties are the relations between nodes. We use special properties of any resource like "label", "abstract" and so on that have been express in DBpedia. Also we use new properties to describe the relations that have not been express in DBpedia for resources, Because we use related concepts to fuzzy logic category by using of web services of [18] and they have no property that show their relation to fuzzy logic category, therefore we need to add new property like "RelatedTo". Indeed we extend our knowledge by adding these relations to it. We save constructed ontology in Owl format. This ontology is a semantic knowledge base which uses global concepts in DBpedia knowledge base. It can be used in other e-learning environments as a common knowledge base. It is an advantage for that and separates it from other databases.

\section{CONCLUSION}

In this article, we tried to present the usage of semantic web techniques in learning systems in order to construct and represent learning knowledge. As it is seen, the produced learning data by the use of DBpedia knowledge base and the semantic web techniques like RDF/XML and OWL format, in addition to syntax, include semantic as well, so it can be shared and reused in learning systems, and intelligent agents can use them in order to exchange information. These are advantages of the proposed method for production of the desired learning data. In the future work we will try to create an efficient and personalized environment by use of intelligent agents and produced knowledge base.

\section{ACKNOWLEDEMENT}

The authors would like to thank the Iranian Telecommunication Center for its financial supports.

\section{REFERENCES}

[1] F. Castro, A. Vellido, À.Nebot, and F. Mugica, "Applying data mining techniques to e-learning problems," Studies in Computational Intelligence (SCI), Vol. 62, pp. 183-221, 2007, Springer-Verlag Berlin Heidelberg.
[2] P. Brusilovsky and C. Peylo, "Adaptive and intelligent web-based educational systems," International Journal of Artificial Intelligence in Education, Vol. 13, no. 2-4, pp. 156-169, 2003.

[3] C. Chen, H. Lee and Y. Chen, "Personalized E-Learning System using Item Response Theory," Computers \& Education, Vol. 44, no. 3, pp. 237-255, 2005.

[4] S. Prakasam and Prof. Dr. R. M. Suresh, "An agentbased Intelligent System to enhance E-Learning through Mining Techniques," (IJCSE) International Journal on Computer Science and Engineering, Vol. 2, no. 3, pp. 759-763, 2010

[5] M. Ueno, "Bayesian Agent in e-learning," E-learning Experiences and Future, Safeeullah Soomro (Ed.), ISBN: 978-953-307-092-6, pp. 129-146, 2010.

[6] F. Ghaleb, et al., "E-Learning Model Based On Semantic Web Technology," International Journal of Computing \& Information Sciences, Vol. 4, no. 2, pp. 63 - 71, August 2006.

[7] T. Berners-lee, J. Hendler and O. Lassila, "The Semantic Web," Scientific American, pp. 34- 43, 2001.

[8] A. Gladun, J. Rogushina, F. Garcia-Sanchez, R. Martinez-Bejar and J. T. Fernandez-Breis, "An Application of Intelligent Techniques and Semantic Web Technologies in E-Learning Environments," Expert Systems with Application, Vol. 36, no. 2, pp. 1922-1931, March 2009.

[9] N. S. Chen, Kinshuk, C. W. Wei, and H. J. Chen, "Mining E-Learning Domain Concept Map from Academic Articles," Computers \& Education, Vol. 50, no. 3, pp. 1009-1021, April 2008.

[10] E. Plotnick, "A graphical system for understanding the relationship between concepts: an ERIC digest," New York: ERIC Clearinghouse on Information and Technology, pp. 1-7, 1997.

[11] M. Brian, "Semantic web Technology", Published in JISC Technology and Standards Watch, pp. 1- 20, 2005.

[12] P. M. Dolia, "Integrating Ontologies into Multi-Agent Systems Engineering (MaSE) for University Teaching Environment," Journal of Emerging Technologies in Web Intelligence, Vol. 2, no. 1, pp. 42- 47, February 2010.

[13] J. Dunkel and R. Bruns, "Semantic E-Learning Agent: Supporting E-learning by semantic web and agents technologies," ENTERPRISE INFORMATION SYSTEMS VI, pp. 237-244, 2006, Springer.

[14] V. N., Convetini, D. Albanese, A. Marengo, V. Marengo and M. Scalera, "The OSEL taxonomy for the classification of learning objects", Vol. 2, pp. 125-138, 2006.

[15] A. Gladun and J. Rogushina, "Use of Ontological Analysis for Student Skills Control in E-Learning: Semantic Web Approach", International Journal of Science and Technology (IJST), Vol. 1, no. 1, pp. 21-31, 2012.

[16] C. Snae and M. Brueckner, "Ontology- Driven ELearning System Based on Roles and Activities for Thai Learning Environment," Interdisciplinary Journal of Knowledge and Learning Objects, Vol. 3, pp. 1-17, 2007.

[17] C. Bizer, et al., "DBpedia - A crystallization point for the Web of Data,", Computer and Information Science, Vol. 7, no. 3, pp. 154-165, 2009, Elsevier. 
[18] R. Mirizzi and T. Di Noia, "From Exploratory Search to Web Search and back", In Proceedings of the Fourth
Ph.D. Workshop in CIKM, PIKM 2010, pp. 1-16, October 2010, Toronto, Canada.

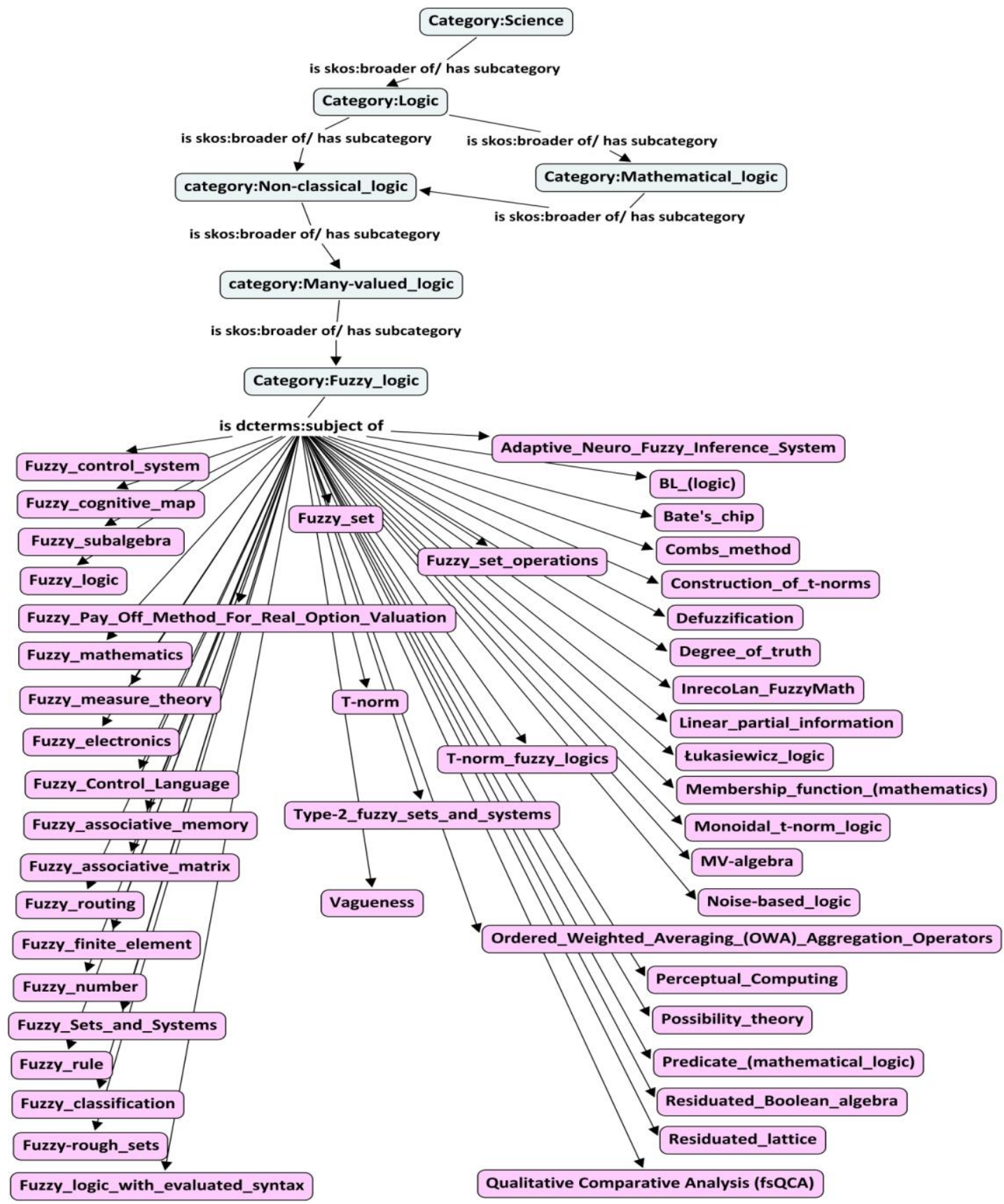

Fig 1: A concept map from fuzzy logic category and its sub-category 